\title{
IDENTIFICAÇÃO DE PROCESSOS EROSIVOS ÀS MARGENS DO RIO MADEIRA (RO/AM) A PARTIR DA INTERFEROMETRIA DIFERENCIAL POR RADAR (DINSAR)
}

\author{
Carlos Tadeu de Carvalho Gamba ${ }^{(a)}$, Rafael Antonio da Silva Rosa ${ }^{(b)}$, João Bosco Nogueira \\ Junior $^{(c)}$ \\ (a) Instituto de Pesquisas Tecnológicas do Estado de São Paulo (IPT), carlosgamba@ipt.br \\ (b) Bradar Indústria S.A., rafael.rosa@bradar.com.br \\ (c) Santo Antônio Energia, joaobosco@santoantonioenergia.com.br
}

\section{Eixo: GEOTECNOLOGIAS E MODELAGEM ESPACIAL EM GEOGRAFIA FÍSICA}

\begin{abstract}
Resumo/
Este trabalho descreve o uso da técnica DInSAR (Interferometria Diferencial por Radar de Abertura Sintética), gerada com a Banda P, e da diferença entre modelos digitais de superfície (DSM), gerada com a Banda X, na detecção de movimentos do terreno causados por processos erosivos nas margens de Rio Madeira. A área de estudo caracterizou-se por um trecho de rio que se inicia no barramento da UHE Santo Antônio, no município de Porto Velho (Rondônia), e se estende até o município de Humaitá (Amazonas). A metodologia foi estabelecida a partir da comparação de séries temporais geradas pelo radar, com medições obtidas em campo. Os resultados apontaram um desempenho positivo desta tecnologia. Acredita-se, entretanto, que a utilização de métodos de processamento mais robustos pode melhorar a qualidade dos resultados. De qualquer forma, constata-se que esta aplicação já apresenta grande potencial para o monitoramento de movimentos da superfície do terreno em larga escala.
\end{abstract}

Palavras chave: sensoriamento remoto, processamento de imagens SAR, interferometria diferencial, DInSAR, processos erosivos.

\section{Introdução}

Como já é de conhecimento, a natureza é um ambiente dinâmico. Muitas vezes, fenômenos capazes de produzir transformações no meio físico ocorrem de maneira lenta e levam milhões de anos para se concretizarem. Em alguns casos, as mudanças podem ser constatadas em questão de meses ou, até, dias. No mundo tropical, entre as alterações do meio que podem ser observadas num curto intervalo de tempo estão os processos erosivos, essencialmente aqueles vinculados aos ciclos hidrológicos. Monitorar tais eventos nem sempre é uma tarefa fácil, em especial quando se trata de conduzir esta ação com o objetivo principal de prevenir alterações bruscas no meio físico que sejam capazes de trazer prejuízos à economia e à sociedade. Um dos fatores que dificultam a realização desta tarefa é a necessidade de monitorar estes fenômenos de perto, o que nos obriga, na maioria das vezes, a instalar uma rede de equipamentos que possa fornecer informações precisas, em tempo real, sobre uma determinada dinâmica permitindo assim a 
tomada de decisões antes da ocorrência de um evento adverso. O sensoriamento remoto, já há um bom tempo, tem sido uma alternativa viável quando se trata de observar movimentos da superfície causados, ou não, por processos erosivos, principalmente devido à sua capacidade de levantar informações sobre áreas mais extensas. A técnica da Interferometria Diferencial obtida por Radar de Abertura Sintética, ou simplesmente DInSAR, foi desenvolvida para efetuar medições de pequenas oscilações da superfície terrestre. Experiências com esta tecnologia, que opera sobre as diferenças de fase entre duas ou mais aquisições feitas por um sensor SAR, acoplado a uma plataforma orbital ou aérea, vem sendo desenvolvidas por vários pesquisadores ao longo dos últimos anos. Trabalhos como os de Lanari et al. (2004), Ferretti et al. (2007), Crosetto et al. (2011) realizados sobre dados SAR gerados nas Bandas C e X, têm demonstrado o potencial desta ferramenta na medição de pequenas movimentações da superfície, em escala centimétrica ou até milimétrica. Mas apesar do uso desta tecnologia ter se expandido de maneira mais pronunciada na Europa a partir dos anos de 1990, em virtude principalmente da preocupação com os movimentos causados por abalos sísmicos, a DInSAR já vem sendo utilizada desde o final da década de 1980 (Gabriel et al., 1989).

No Brasil, contudo, em detrimento do pioneirismo estabelecido com o uso de um SAR aerotransportado, no levantamento de informações da superfície, realizado dentro dos projetos RADAM e RADAMBRASIL, as aplicações com a DInSAR são recentes. Experimentos realizados por Mura et al. (2015) e Paradella et al. (2015) entre outros, a partir de dados obtidos por um SAR interferométrico que trabalha com a Banda X, foram iniciados no início da década de 2010 com o intuito de monitorar a estabilidade de taludes e as deformações superficiais na Mina de Ferro de Carajás, no Estado do Pará, e constataram a eficiência deste recurso tecnológico na medição de movimentações da superfície em um ambiente tropical. Entretanto, aquisições de radar feitas a partir de plataformas orbitais, operando nas Bandas X e C, não só podem apresentar limitações em razão de interferências causadas pela atmosfera (Zebker et al., 1997), como também podem se mostrar inadequadas para algumas aplicações, por não permitirem a obtenção de informações da superfície do terreno quando a área de interesse encontra-se coberta por uma densa cobertura florestal, condição que pode ser superada quando se utiliza radares que trabalham com as Bandas P ou L (Macedo et al., 2012; Jones et al., 2012). Essa possibilidade de penetração das ondas do radar abaixo da estrutura do dossel torna-se um diferencial importante pois permite, além de levantamentos topográficos precisos, a medição de processos erosivos e de sedimentação que ocorrem no interior da mata (Macedo e Wimmer, 2015).

O objetivo deste trabalho foi avaliar o potencial da técnica DInSAR, gerada a partir de aquisições sucessivas com um SAR aerotransportado operando com a Banda P, e da subtração entre modelos digitais de superfícis (DSM), gerados com a Banda X, na identificação e quantificação de processos erosivos de 


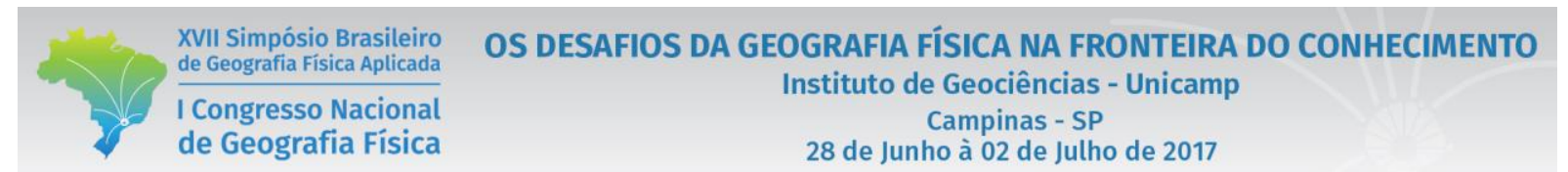

margem de rio caracterizados basicamente pela movimentação vertical do terreno. $\mathrm{O}$ trecho escolhido para este estudo (Figura 1) compreende uma faixa marginal que se estende por aproximadamente $240 \mathrm{~km}$ ao longo do rio Madeira em direção à jusante, a partir da barragem da UHE Santo Antônio, no município de Porto Velho (RO), até o município de Humaitá (AM). A aquisição e o processamento dos dados de radar bem como as atividades de campo foram realizados entre os meses de agosto de 2015 até julho de 2016.

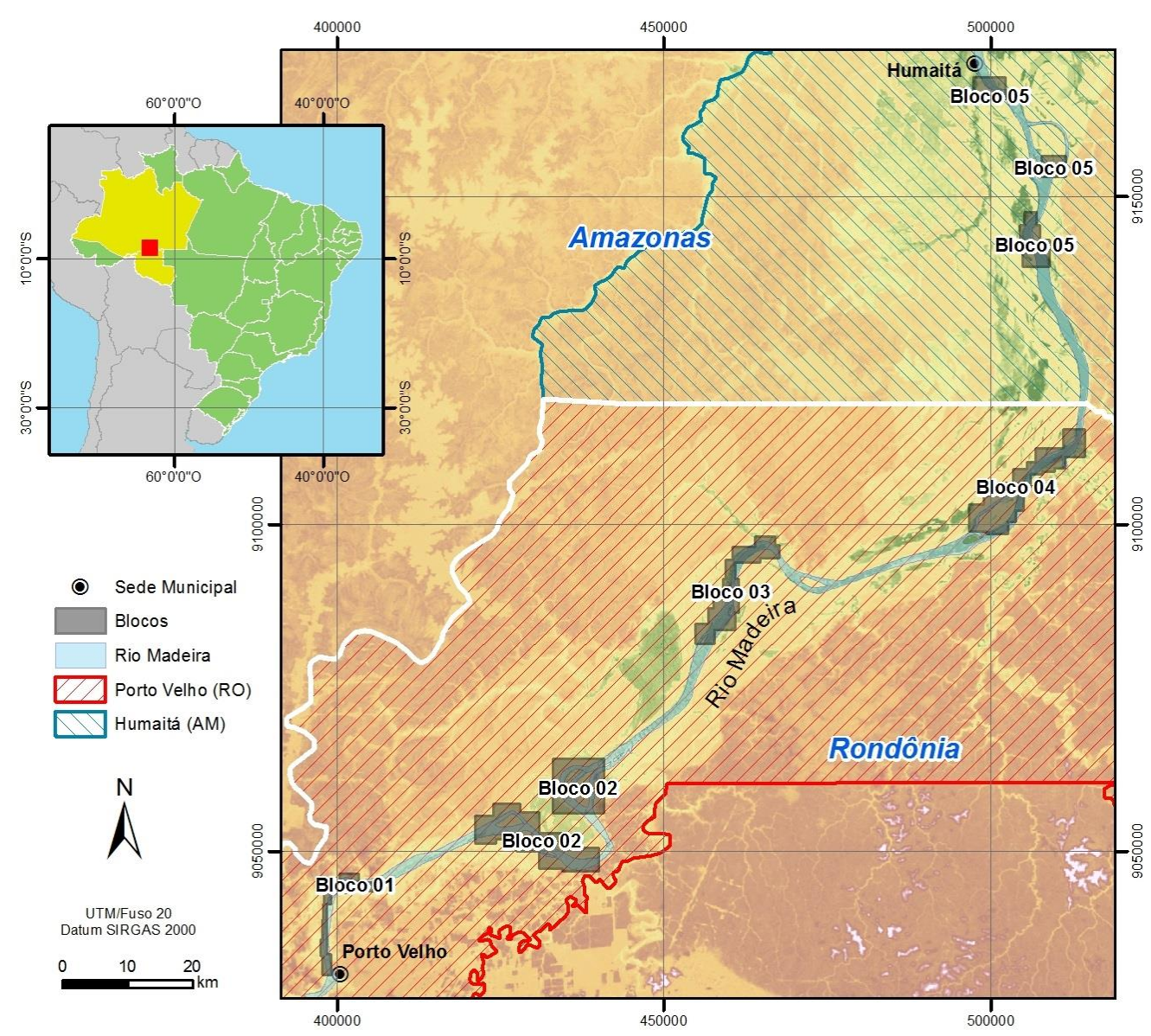

Figura 1 - Localização da área de estudo com a delimitação dos blocos de amostragem.

\section{Procedimentos metodológicos}

\subsection{Aquisição dos dados SAR interferométricos}

A aquisição de dados SAR foi concretizada a partir de 11 voos de coleta, distribuídos ao longo de 12 meses, com intervalos mensais. A definição deste período de tempo teve como intuito principal, a cobertura de um ciclo hidrológico anual completo, englobando os períodos de seca (vazante) e de chuva (enchente). Essa série temporal mais longa também foi necessária para garantir a qualidade das análises realizadas neste 


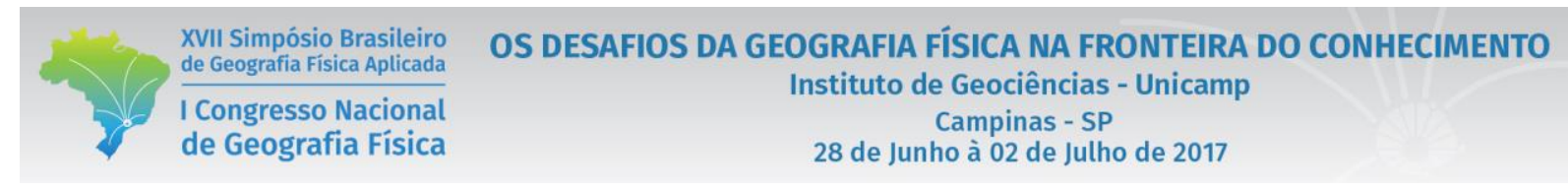

estudo, pois com a obtenção de uma sequência multitemporal mais extensa, foi possível evitar que o uso da técnica DInSAR fosse rejeitado por falta de um número suficiente de dados coletados. Outra particularidade relativa à aquisição dos dados SAR diz respeito à dinâmica dos processos: como se trata da detecção de mudanças de magnitude centimétrica, intervalos de coleta muito longos poderiam acarretar em medições cujos valores superam àquelas estabelecidas no escopo do projeto. Com a limitação dos voos de coleta a intervalos mais restritos, próximos a um mês, este risco foi eliminado.

O primeiro voo de aquisição, denominado de etapa de mapeamento, foi realizado antes das medições serem iniciadas, em um curto período, onde não houve tempo para a ocorrência de movimentações do terreno. Esta etapa requisitou uma maior cobertura de linhas de voo do que as aquisições subsequentes (Figura 2). Neste mapeamento, tanto os dados interferométricos da Banda $\mathrm{P}$, quanto da Banda $\mathrm{X}$, foram utilizados para gerar uma série de produtos caracterizados por imagens de amplitude na Banda $\mathrm{X}$, por modelos digitais de terreno (DTM), gerados a partida Banda P e por modelos digitais de superfície (DSM), gerados na Banda X. A confecção dos modelos digitais (DTM e DSM) possibilitou a obtenção de informações detalhadas a respeito da topografia local: o DSM apresenta os valores altimétricos de tudo que esteja presente na superfície; e o DTM descreve as informações topográficas daquilo que se encontra abaixo da vegetação.
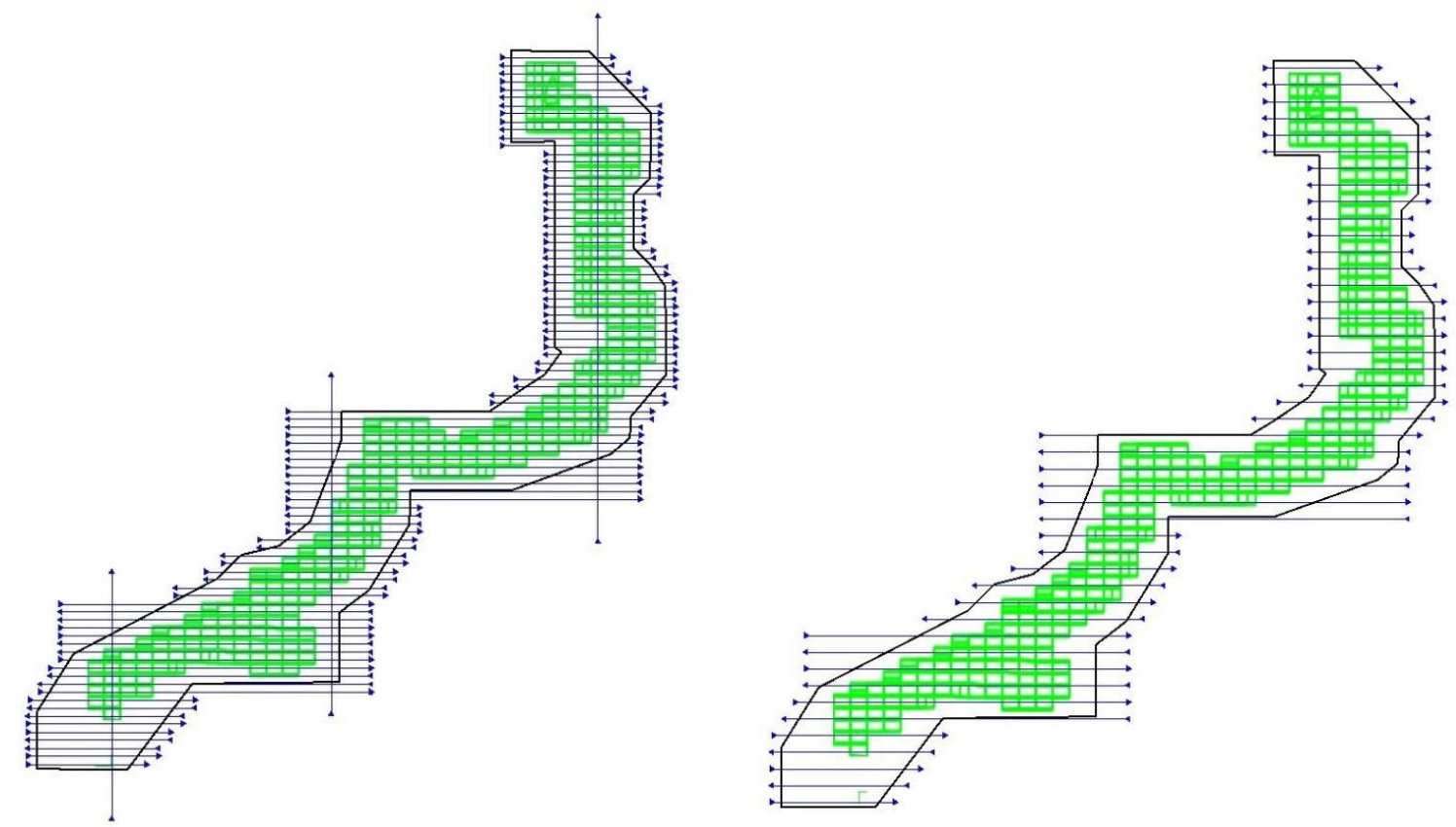

Figura 2 - Planos de voo para o mapeamento (à esquerda) e para o monitoramento dos processos (à direita).

Entretanto, apesar destes dois produtos fornecerem informações do terreno com alta resolução espacial, a aquisição de ambos foi motivada por diferentes questões. No caso do DTM, o objetivo principal foi gerar um 
conjunto de dados necessários à execução da filtragem da componente topográfica da fase. Em outras palavras, sua geração foi realizada para separar perfeitamente os dados provenientes da topografia, dos dados provenientes de movimentações ocorridas neste terreno, garantindo assim a precisão desejada durante o processamento das informações destinadas à aplicação da técnica DInSAR. O DSM foi gerado para se detectar mudanças de ordem macrométrica, quando o volume erodido produz alterações mais pronunciadas no terreno. Nesse caso, por não dependerem da coerência temporal dos dados SAR, as mudanças podem ser constatadas pela simples comparação entre modelos digitais de superfície. Com isso, a combinação entre DInSAR e diferença entre modelos digitais de superfície possibilitou que fossem detectados e quantificados, fenômenos geodinâmicos com diferentes ordens de grandeza.

As séries temporais foram obtidas a partir de um número maior de aquisições (long-term interferogram) e foram realizadas após o voo de mapeamento, em um momento onde os eventos de movimentação do terreno passaram a ser factíveis. Essas aquisições sucessivas conhecidas como long-term permitem a análise temporal dos dados DInSAR, viabilizando o modelamento e a validação das medidas.

Os dados SAR na Banda $\mathrm{P}$ foram adquiridos em linhas de voo com altíssimo rigor de posicionamento. A plataforma aérea repetiu os voos com um desvio de visada de no máximo 5 metros e variação de squint de mais ou menos 2 graus. A posição da aeronave foi corrigida em tempo real com a ajuda de um sistema GPS/INS (Sistema de Navegação Inercial) a bordo (Macedo e Scheiber, 2005, Macedo et al., 2012). Já, a posição e a altitude das antenas do radar, durante o voo, foram determinadas pelo sistema IMU/DGPS (Unidade de Medição Inercial e GPS Diferencial). Este sistema pós-processado é capaz de garantir uma precisão rotacional de 0,1 grau e uma precisão translacional na ordem de 1,0 decímetro. Mesmo assim, devido principalmente às condições atmosféricas, é impossível que o avião voe em uma linha reta perfeita. Por isso, o processamento dos dados SAR foi realizado com dois tipos de compensações de movimento: uma Compensação de Movimento padrão (MoCo); e uma compensação de movimento dependente de topografia precisa e da abertura da antena (PTA-MoCo).

\subsection{Processamento dos dados SAR e geração das séries temporais}

Após a coleta de dados foi implementado um corregistro entre as duas aquisições, da ordem de subpíxels, para colocá-las exatamente sob a mesma geometria, com a ajuda de um modelo de elevação proveniente do mesmo sistema de radar no qual os dados foram adquiridos. Em seguida foi aplicada uma filtragem para diminuição de ruído e uma compensação de erros residuais do posicionamento das antenas. Este procedimento fez com que os modelos de elevação não apresentassem nenhuma ondulação 


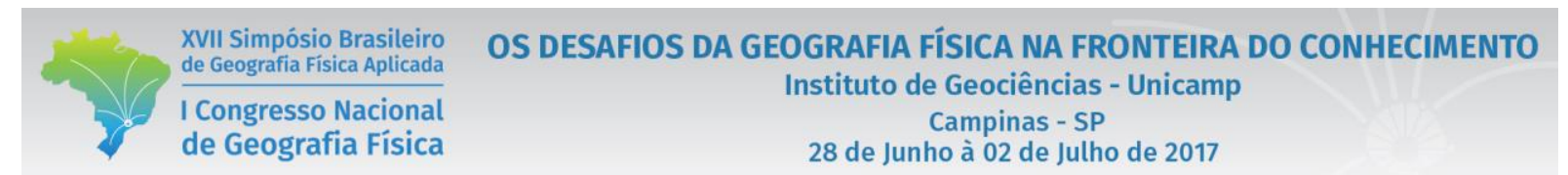

significante. A série temporal DInSAR foi gerada a partir de dados SLC (single-look complex) com 1,5 metro de resolução espacial.

A Figura 3 apresenta o fluxograma da metodologia de processamento aplicada neste trabalho para se alcançar os resultados esperados. Este processamento teve como principal objetivo permitir que o sistema disponibilizasse uma série temporal, com os dados de radar, que permitisse estimar o movimento acumulado do terreno, a partir da integração das informações provenientes de uma aquisição sucessiva de interferogramas (Macedo e Wimmer, 2015). Distintamente de como está proposto em Macedo et al. (2012), a análise desta série aplicada aos dados interferométricos foi transferida para o final do diagrama em virtude da praticidade e da rapidez que esta mudança possibilita.

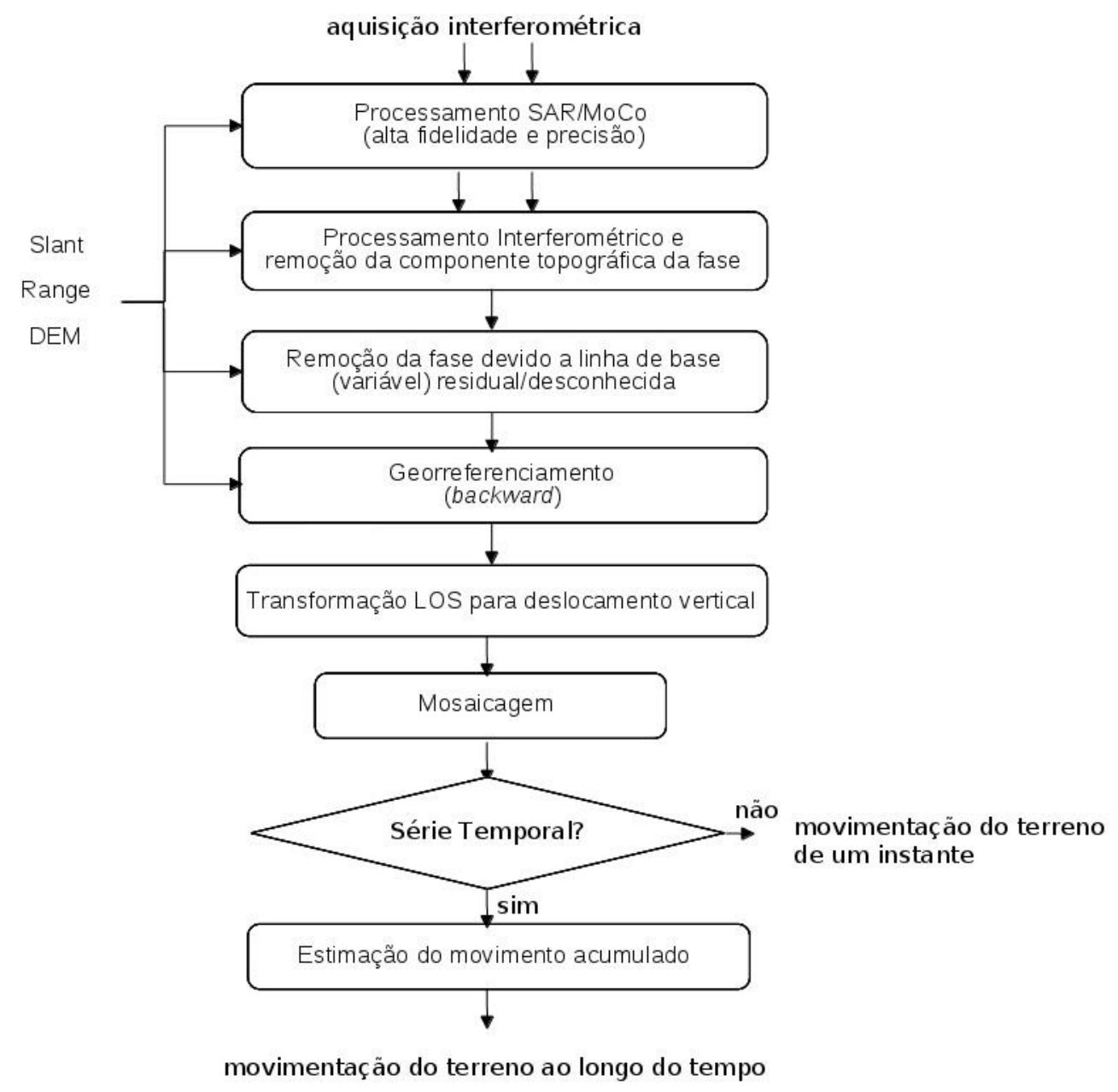

Figura 3 - Fluxograma da metodologia de processamento dos dados SAR interferométricos.

Como também se espera que haja perda de coerência ao longo do tempo, entre uma aquisição e outra de dados SAR, foi aplicada uma filtragem simples, caracterizada pela repetição do último valor de fase da série 
que possua coerência. Este procedimento garantiu a continuidade do processo e o acompanhamento da tendência dos movimentos.

A série temporal dos modelos digitais de superfície (DSM), gerados com a Banda X, foi realizada a partir do cálculo da diferença entre o primeiro DSM obtido em agosto de 2015, durante a aquisição de mapeamento, e os demais, coletados mês a mês até julho de 2016. Como já abordado, esta técnica fornece informações quantitativas sobre mudanças topográficas ocorridas na superfície, em essência, processos erosivos de grande magnitude associados ao canal fluvial, que se caracterizam por alterações abruptas na superfície: desmatamentos, crescimento da vegetação, movimentos de volumes consideráveis de terra; que fazem com que ocorra perda por completo da coerência, entre as sucessivas aquisições interferométricas do radar, impossibilitando assim a aplicação da técnica DInSAR. A utilização desta diferença entre os DSM foi importante para ajudar na determinação, compreensão e identificação de eventos erosivos ou de outros processos geodinâmicos detectáveis através da DInSAR.

A hipótese levantada neste trabalho foi de que alterações no DSM ao longo das margens do rio referem-se a desbarrancamentos ou processos de sedimentação. Enquanto que a acurácia obtida com a DInSAR, é da ordem de centímetros, com esta técnica, a detecção dos movimentos foi estimada em $2 \mathrm{~m}$, considerando-se que o erro quadrático médio (RMSE) de cada DSM é de $1 \mathrm{~m}$.

\subsection{Análise e validação de campo das movimentações estimadas pelo radar}

As etapas de campo introduzidas neste trabalho tiveram por finalidade a obtenção de medidas in situ que pudessem ser comparadas com aquelas estimadas pelo SAR interferométrico. Estas observações foram orientadas em dois caminhos distintos: um deles direcionado a obter registros de grandes movimentos ocorridos na margem, para que tais informações pudessem ser comparadas posteriormente com as movimentações observadas na diferença entre os modelos digitais de superfície (DSM); e outro, destinado à obtenção de medidas de pequenas movimentações da superfície, com o intuito de verificar se a técnica DInSAR é capaz de aferir com precisão estas informações.

Para realizar estas medidas foram priorizados os trechos de margem, não só pelo fator logístico, em razão da extensão da área a ser monitorada, mas também pela natureza dos fenômenos a serem amostrados. Os sistemas fluviais, além de funcionarem como canais de escoamento, exercem um forte controle sobre o transporte de sedimentos e condicionam sobremaneira a ação erosiva dos rios sobre o substrato geológico ao longo do tempo. As margens acabam se tornando mais sensíveis aos processos erosivos por estarem sujeitas à alteração dos gradientes hidráulicos causada pela oscilação do nível do freático durante os sucessivos ciclos de enchente e vazante do rio. Esta dinâmica hidrológica natural, que pode ser 
potencializada durante a operação de um reservatório, faz com que estes compartimentos sofram com o aumento da pressão erosiva nas bordas do canal durante as cheias e com o aumento do escoamento, tanto em superfície quanto em subsuperfície, nas vazantes. Conjugados, estes processos podem ocasionar solapamentos de terra nas bordas do canal, que em muitos casos representa o aumento dos riscos às populações ribeirinhas.

Para monitorar a movimentação das margens do Rio Madeira, os procedimentos utilizados basearam-se em Fernandez (1990) e Siqueira et al. (2013) e consistiram na instalação de 202 estacas ao longo das margens, distribuídas em 20 áreas de interesse, divididas em 5 blocos, totalizando um montante de 101 seções de medição. Os pontos de medição foram escolhidos com base na análise da dinâmica fluvial observada em imagens aéreas do Google Earth ${ }^{\circledR}$ e dos satélites Landsat 5 e 8 nos anos de 2001, 2007 e 2014. As estacas, feitas em madeira, foram instaladas em pares, perpendicularmente às margens. Em algumas seções foram instalados marcos de concreto, além da segunda estaca, para auxiliar na posterior localização das mesmas, caso fossem perdidas e/ou removidas. Cada par foi organizado com uma estaca para a medição (chamada E), colocada próxima à margem, e outra que serviu de referência (chamada P) e foi instalada a uma distância de 15 a 25 metros para além da estaca de medição, em um terreno mais estável.

As estacas foram identificadas a partir das informações dos respectivos blocos e áreas, sendo nomeadas com o prefixo $\mathrm{P}$, quando utilizadas como referência e $\mathrm{E}$, quando utilizadas para medição. $\mathrm{O}$ georreferenciamento das mesmas foi obtido a partir de um GNSS diferencial (Trimble Pro XRT), com correção da posição em tempo real por meio da rede OmniSTAR. Posteriormente, com o intuito de melhorar a acurácia dos posicionamentos, foi feita a correção dos arquivos GNSS com informações levantadas a partir de estações base da RBMC do IBGE localizadas em Porto Velho (RO) e Humaitá (AM), o que proporcionou uma precisão em torno de $40 \mathrm{~cm}$ aos dados planimétricos. O desnível altimétrico $(\Delta \mathrm{H})$ entre a estaca próxima à margem $(\mathrm{E})$ e a estaca de referência $(\mathrm{P})$, que caracterizou a magnitude do movimento, foi estabelecido com precisão milimétrica por meio de medições efetuadas com um Nível Eletrônico Sprinter 250M da Leica Geosystems.

$\mathrm{O}$ monitoramento das áreas acompanhou o período de aquisição de dados SAR, sendo realizado entre os meses de agosto de 2015 e julho de 2016. Apesar dos imageamentos serem realizados mês a mês, o intervalo entre uma medida de campo e a seguinte foi de dois meses. Ainda assim, em todas as campanhas, as atividades de medição foram executadas simultaneamente à aquisição dos dados SAR, considerando apenas uma pequena defasagem temporal em razão da velocidade com que as informações foram coletadas pela aeronave. Essa estratégia permitiu acompanhar os processos de movimentação das margens durante a evolução do ciclo de enchente e vazante do rio. As campanhas de campo (um total de 


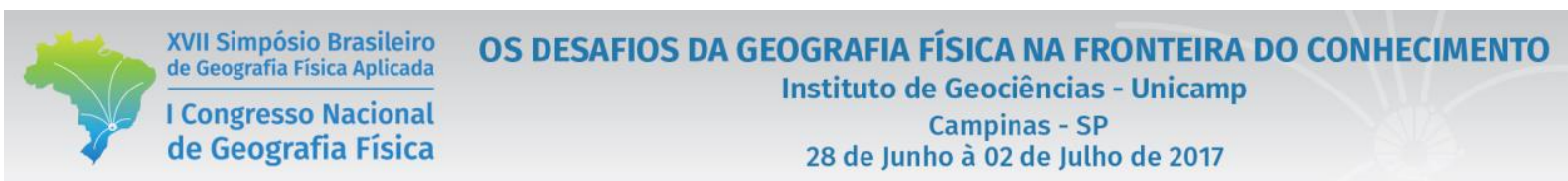

6) foram divididas em etapas de instalação ( $1^{\mathrm{a}}$ etapa $)$ e de medição ( $2^{\mathrm{a}}$ a $6^{\mathrm{a}}$ etapa). Ao final, os dados foram tabulados, revisados e então comparados com os dados de radar.

\section{Discussão dos resultados}

Os resultados obtidos permitiram estabelecer conclusões importantes a respeito do uso da técnica DInSAR e da diferença entre os DSM na identificação e quantificação de movimentações do terreno causadas por processos erosivos. Em ambos os casos, os dados obtidos pelo SAR interferométrico foram capazes de indicar corretamente, na maioria dos casos, a tendência do movimento. Tal movimento tanto foi constatado na diferença entre os DSM quanto na aplicação da técnica DInSAR (Figura 4), foco principal desse trabalho.
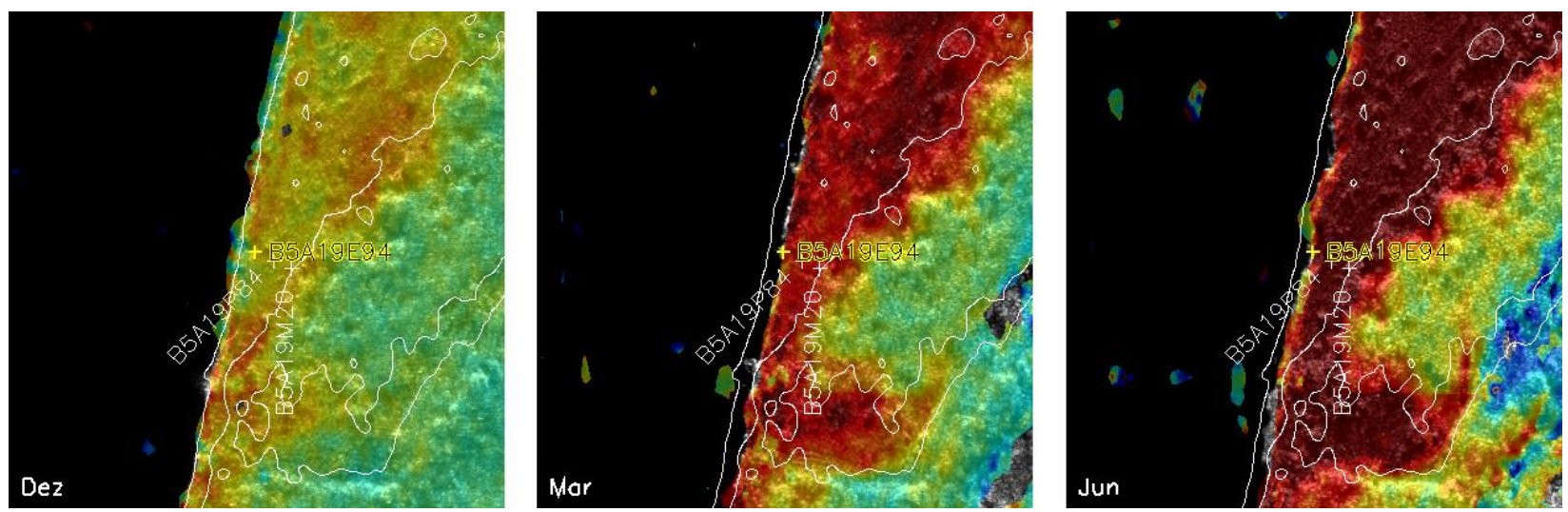

Figura 4 - Série temporal da técnica DInSAR mostrando a evolução da dinâmica do movimento. Os tons avermelhados indicam movimentos descendentes da superfície em escala centimétrica. A cruzeta amarela indica a posição da estaca de medição.

No caso da DInSAR, os resultados atestaram que de um total de 405 medidas efetuadas para toda a série temporal (de agosto de 2015 a julho de 2016), 71\% das movimentações indicadas pelo radar foram no mesmo sentido do movimento constatado em campo. Nesta análise foram consideradas as indicações positivas, dentro do desvio padrão, para os movimentos verticais de subida ou descida da superfície. Ou seja, foi considerado acerto quando o mesmo vetor de movimento foi constatado tanto no dado estimado pelo radar quanto na medida de campo. Os resultados obtidos com a diferença entre os DSM, apesar de não quantificados pelo método das estacas, também mostraram a mesma tendência. As séries temporais geradas com esta técnica também permitiram acompanhar com precisão a evolução do ciclo hidrológico no canal fluvial. Nas imagens do radar é possível observar o momento em que o rio atinge o seu nível máximo (abril) e, posteriormente, com rebaixamento das águas (julho), quando ocorre o recuo da margem 


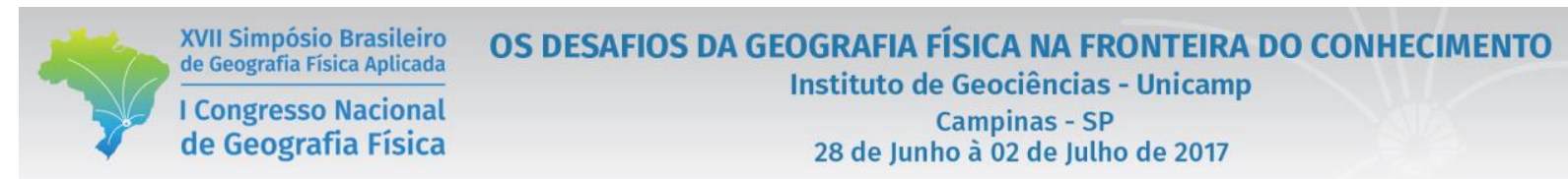

(Figura 5). Neste caso, a estaca de medição foi perdida durante a ação erosiva das águas do rio Madeira entre os meses de dezembro e abril.
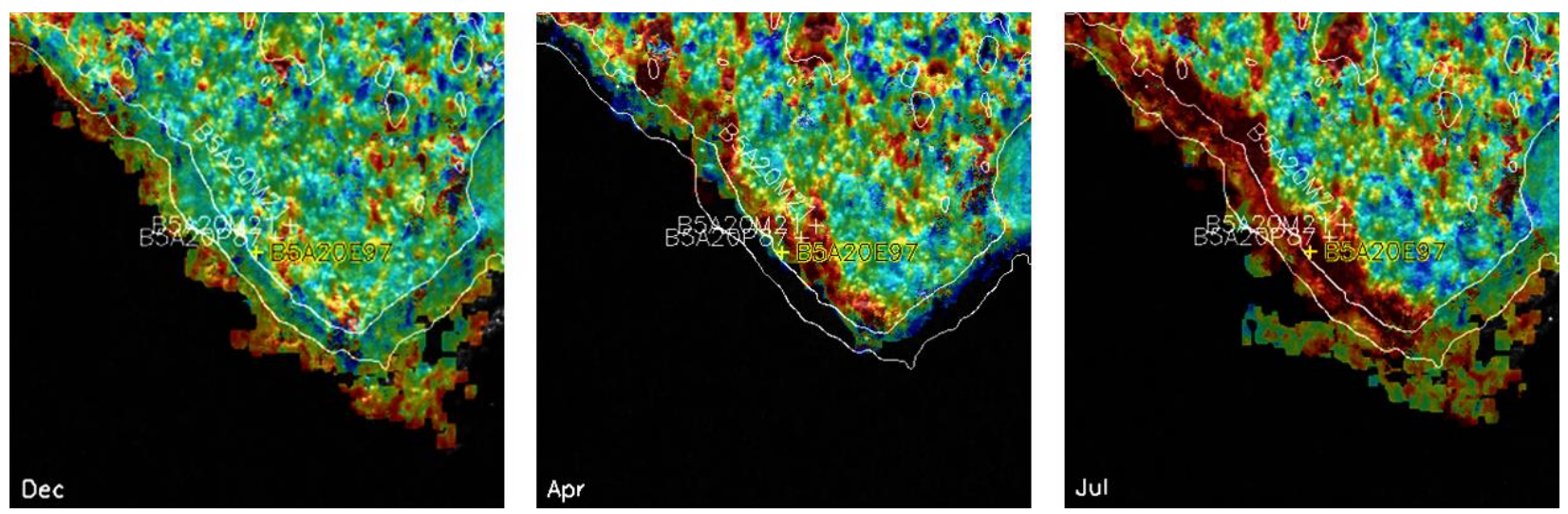

Figura 5 - Série temporal da técnica da diferença entre os DSM. É possível ver o avanço da água (tonalidade escura) sobre as áreas emersas (tonalidades coloridas). Os tons avermelhados indicam movimentos descendentes de terra.

No que diz respeito às comparações estabelecidas entre as medidas coletadas em campo e aquelas estimadas a partir da técnica DInSAR, o desempenho do radar foi menos eficiente. As comparações foram feitas de modo direto, apenas com a implementação de alguns ajustes destinados a se estabelecer um limiar de acerto, pois devido à própria natureza do sistema é impossível trabalhar com comparações absolutas. Na Banda P, por exemplo, interferências causadas por mudanças de umidade podem provocar alterações no sinal resultante do radar, fazendo com que a coerência interferométrica caia para um coeficiente menor que 0,3 (considerando uma escala de valores entre 0 e 1). Este fator de correlação implica um desvio padrão da ordem de $3 \mathrm{~cm}$. Assim, com a finalidade de evitar a incorporação de resultados não confiáveis foi adotado um desvio padrão de $3 \mathrm{~cm}$ como margem de erro (que estima uma diferença da ordem de $6 \mathrm{~cm}$ ) para as medidas produzidas pela DInSAR. Dentro deste limiar, para as 405 medidas efetuadas, verificou-se um acerto de $45 \%$.

Entretanto, tais resultados requerem uma abordagem mais aprofundada da questão. Quando se compara a série histórica nas imagens DInSAR com os resultados das medições de campo, verifica-se que mesmo quando as medidas não estão próximas é possível observar, em vários exemplos, que o radar registra a mesma tendência do movimento observada em campo (Figura 6). Um dos motivos que pode ter relação direta com esta diferença de magnitude, diz respeito à filtragem executada com uma máscara de 17 x 17 metros. Enquanto as medidas de campo são pontuais, as do radar são estimadas em uma área, onde podem ocorrer eventos distintos daqueles ocorridos no local onde a estaca de medição foi instalada. Por este 


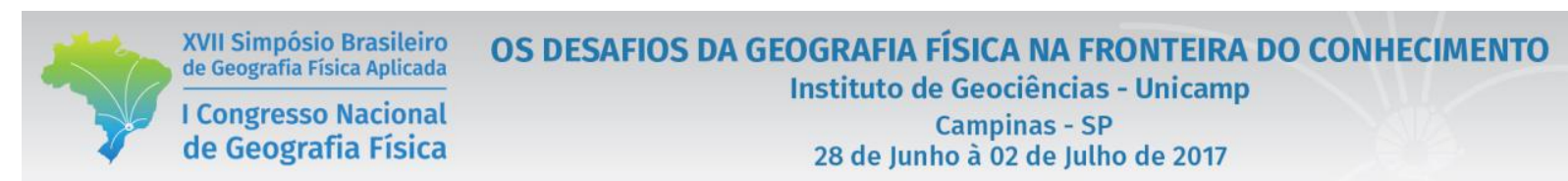

motivo, é de se esperar que tais eventos exerçam algum tipo de influência sobre os resultados da medição estimados pela técnica DInSAR. Outro fator que pode interferir nas medidas de campo é a instabilidade de algumas estacas de referência causada pela magnitude dos processos erosivos.
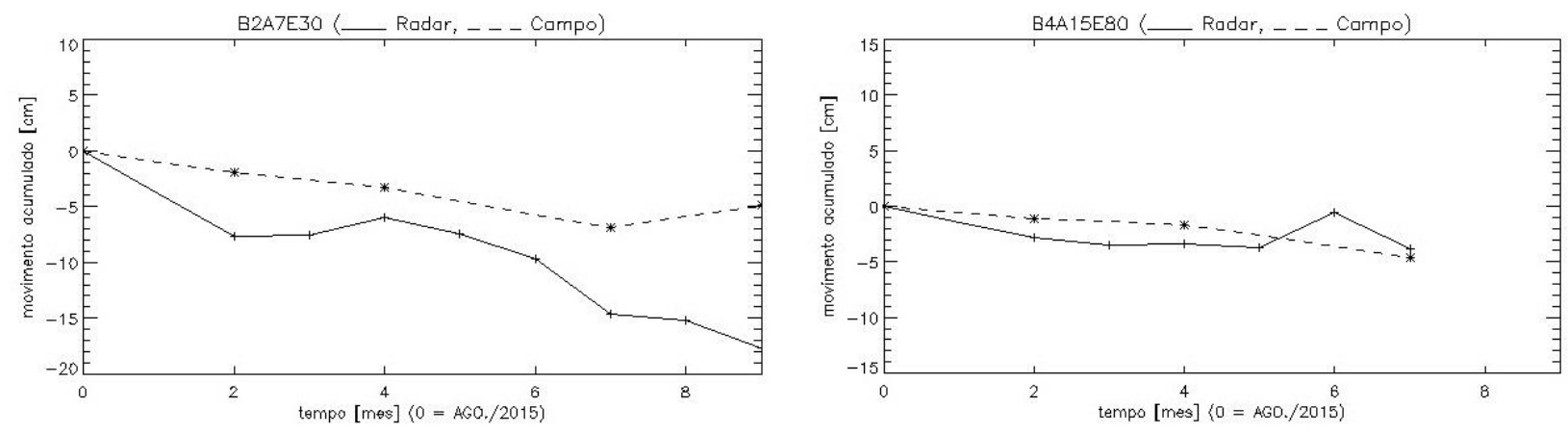

Figura 6 - Comparações entre as medidas obtidas com a DInSAR e pelo trabalho de campo.

Experimentos realizados com uma janela de filtragem menor ( 5 x 5 metros), apresentaram melhor correlação entre as medidas de campo e do radar. Ainda assim, o consequente aumento do ruído nos dados SAR não permitiu estabelecer conclusões definitivas a respeito desta questão. Uma alternativa que vem sendo avaliada é a aplicação de um processo de filtragem mais robusto, uma vez que questões relacionadas às diferenças de magnitude podem, também, estar vinculadas à propagação do ruído ao longo da série temporal. Isto explicaria, em parte, o fato das diferenças aumentarem conforme o tempo avança.

\section{Conclusões}

É possível concluir, a partir da análise de séries temporais obtidas com a técnica DInSAR e com a diferença entre modelos digitais de superfície (DSM), que dados interferométricos gerados nas Bandas X e P, por um SAR aerotransportado, são suficientemente sensíveis e precisos para identificar movimentos do terreno causados por processos erosivos de margem de rio. Os resultados também permitem concluir que o ambiente tropical úmido e a vasta cobertura por vegetal não constituem empecilhos para a utilização desta tecnologia.

Em um universo de 405 medições realizadas em 101 seções de medição, localizadas em situações de mobilidade distintas, obteve-se com a técnica DInSAR um acerto de $71 \%$ no sentido do movimento vertical e de $45 \%$ na magnitude destas medidas em comparação com aquelas obtidas em campo. O uso dos modelos digitais de superfície (DSM), além de constituírem uma importante referência topográfica, também demonstrou um alto potencial de aplicação na detecção de movimentos do terreno e na identificação de alterações das linhas de margem do canal fluvial. 


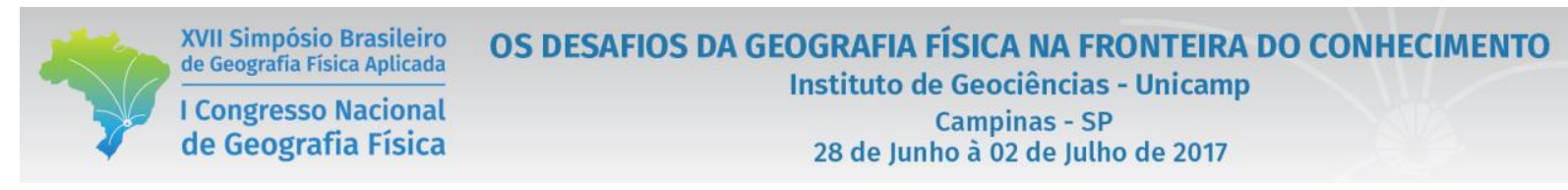

Entende-se que as conclusões a respeito das diferenças entre a magnitude das medidas obtidas em campo e aquelas estimadas pelo radar, bem como a correlação entre as mesmas, carecem de aprofundamento. Melhorias adicionais à cadeia de processamento, como a geração de redes de redundância de interferogramas e filtros de maior resolução espacial estão sendo testados. Pretende-se também analisar o potencial do uso conjunto dos dados DInSAR e DSMs, na compreensão e na classificação dos processos de erosão estudados. De qualquer forma já é possível concluir que os dados do SAR interferométrico aqui utilizados são capazes de apontar, com eficiência, a tendência de movimento da superfície.

\section{Bibliografia}

CROSETTO, M.; MONSERRAT, O.; CUEVAS, M.; CRIPPA, B. Spaceborne differential SAR interferometry: Data analysis tools for deformation measurement. Remote Sensing, v. 3, n. 2, p. 305-318, 2011.

FERNANDEZ, O. V. Q. Mudanças no canal fluvial do rio Paraná e processos de erosão nas margens. Região de Porto Rico, PR. 1990. 86 p., 86p. Dissertação (Mestrado), IGCE/UNESP, Rio Claro, 1990.

FERRETTI, A.; SAVIO, G, BARZAGHI, R.; BORGHI, A.; MUSAZZI, S.; NOVALI, F.; PRATI, C.; ROCCA, F. Submillimeter accuracy of InSAR time series: Experimental validation. IEEE Transactions on Geoscience and Remote Sensing. v. 45, n. 5, p. 1142-1153, 2007.

GABRIEL, A.K., GOLDSTEIN, R.M., ZEBKER, H.A. Mapping small elevation changes over large areas: differential radar interferometry. Journal of Geophys. Research: Solid Earth, v. 94, n. B7, p. 9183-9191, 1989.

JONES, C. E.; BAWDEN, G.; DEVEREL, S.; DUDAS, J.; HENSLEY, S.; YUN, S. H. Study of movement and seepage along levees using DINSAR and the airborne UAVSAR instrument. In: SPIE Remote Sensing. International Society for Optics and Photonics, 2012. Edinburgh, U.K., p. 85360E-85360E-8.

LANARI, R., MORA, O., MANUNTA, M., MALLORQUí, J.J., BERARDINO, P., SANSOSTI, E. A smallbaseline approach for investigating deformations on full-resolution differential SAR interferograms. IEEE Transactions on Geosciences and Remote Sensing, v. 42, n. 7, p. 1377-1386, 2004.

MACEDO K. A. C.; SCHEIBER, R. Precise topography-and aperture-dependent motion compensation for airborne SAR. IEEE Geoscience and Remote Sensing Letters, v. 2, n. 2, p. 172-176, 2005.

MACEDO K. A. C.; WIMMER, C.; BARRETO, T. L.; LUBECK, D.; MOREIRA, J. R.; RABACO, L. L. M.; OLIVEIRA, W. J. Long-term airborne DInSAR measurements at $X$-and P-Bands: A case study on the application of surveying geohazard threats to pipelines. IEEE Journal of Selected Topics in Applied Earth Observations and Remote Sensing, v. 5, n. 3, p. 990-1005, 2012. 
MACEDO K. A. C.; WIMMER, C. Time series of airborne DInSAR data over the Amazon flooded vegetation: Water level changes. In: IEEE International Geoscience and Remote Sensing Symposium, 2015 (IGARSS). Proceedings. 2015, Milan, Italy, p. 5252-5255.

MURA, J. C., PARADELlA, W. R., GAMA, F. F., DOS SANTOS, A. R., \& SILVA, G. G. Monitoramento de deformações de superfície em mina de ferro a céu aberto (N5W-Carajás) utilizando série temporal de Interferometria Diferencial SAR (DInSAR), baseado em dados do satélite TerraSAR-X. In: Simpósio Brasileiro de Sensoriamento Remoto (SBSR), 17, 2015, João Pessoa. Anais... São José dos Campos: INPE, 2015, p. 911-918.

PARADELLA, W. R.; FERRETTI, A.; MURA, J. C.; COLOMBO, D.; GAMA, F. F.; TAMBURINI, A.; SANTOS, A. R.; NOVALI, F.; GALO, M.; CAMARGO, P. O.; SILVA, A. Q.; SILVA, G. G.; SILVA, A.; GOMES, L. L. Mapping surface deformation in open pit iron mines of Carajás Province (Amazon Region) using an integrated SAR analysis. Engineering Geology, v. 193, p. 61-78, 2015.

SIQUEIRA, A. G.; AZEVEDO, A. A.; DE SOUZA, L. A. P.; SILVA, M. Modificações fluviais à jusante de barragens. In: $14^{\circ}$ Congresso Brasileiro de Geologia de Engenharia e Ambiental, 14, 2013, Rio de Janeiro, Brasil, (s.n.).

ZEBKER, H. A.; ROSEN, P. A.; HENSLEY, S. Atmospheric effects in interferometric synthetic aperture radar surface deformation and topographic maps. Journal of Geophysical Research: Solid Earth, v. 102, n. B4, p. 7547 7563, 1997. 